\title{
Eat MAC Diet! -That ensure Microbial Health and thereby Your Own Health!
}

\section{Balasubramanyam*}

Dean of Research Studies and Senior Scientist, Madras Diabetes Research Foundation, Chennai, India

*Corresponding Author: M Balasubramanyam, Dean of Research Studies and Senior Scientist, Madras Diabetes Research Foundation, Chennai, India. E-mail: baluglobaldiab@gmail.com

Received: June 24, 2019; Published: July 08, 2019

DOI: $10.31080 /$ ASNH.2019.03.0360

Increasing evidence indicates that modern lifestyle and diet transition has led to a substantial depletion of the human gut microbiome. While this microbiome depletion is linked to the rampant increase of several chronic diseases, there is a paradigm shift in human nutrition towards 'microbiome-focused precision or personalized nutrition'. Diet indeed, plays an important role in shaping the structure and function of the gut microbiota. We are what we eat and that's why everyone is prescribed for healthy food choice. Do you know the food we eat plays an essential role in maintaining the richness [1] and function of our gut microbiota (trillions of bacteria that live within our digestive tract) and there by dictates our health and keeps us disease-free?

Want to be Healthy? - Choose MAC diet

Much of the carbon and energy for members of the microbiota originate from plant- and animal-derived dietary carbohydrates and plant fibres (resistant to degradation and absorption by the host). Microbial competition within the gut is intense for metabolic access to the energy and carbon sequestered in these molecules. The complex carbohydrate portion of dietary fiber that can be metabolized by gut microbes, were proposed to be referred by the term "microbiota-accessible carbohydrate" (MAC) [2]. MACs are complex carbohydrates, the types found in fruits, vegetables, whole grains, and legumes. MACs serve as selective agents, altering the composition of the microbiota, but also dictate the functionality and metabolic output. Short-chain fatty acids (SCFAs), namely, acetate, butyrate, and propionate, are released by gut bacteria during fermentation of dietary fibers; they operate through specific receptors on the host and offer several metabolic benefits. When we adapt to MAC-deprived diet, we also lose the metabolic benefits of gut-microbiome derived metabolites. While Clostridium difficile (Cd) is an opportunistic diarrheal pathogen and Cd infection (CDI) represents a major healthcare concern, recent studies revealed that CDI can be suppressed through the addition of either a diet containing a complex mixture of MACs or a simplified diet containing inulin as the sole MAC source [3].

It's a fact - Modern Society consume MAC-deprived diet

Modern diet consists of heavily processed foods, rich in fat, sugar, protein, and a variety of additives, while remaining low in micronutrients and dietary fiber. The recommended daily intake of dietary fiber [4] is at least $30 \mathrm{~g}$, although, on average, those on the Western diet only consume $15 \mathrm{~g}$. People in traditional societies (for example, Hadza hunter-gatherers of Tanzania) where fiber intake can reach 50-120 g/day, are associated with a much more diverse gut microbiota when compared with people in Western countries [5]. A diverse microbiome (microbiome richness) is associated with "good health," while low diversity and micrbiome dysbiosis have been correlated to several non-communicable diseases including type 2 diabetes.

Is our microbiome paradise lost?

Low consumption of MACs over generations has been shown to result in complete disappearance of beneficial bacterial strains in a preclinical mice model study [6]. If we extrapolate this finding, this seems to suggest that long-term-reduced MAC consumption over generations will likely also have detrimental effects in humans. This might be true for developing countries like India in the diet transition where we might have lost our microbiome paradise! The message is clear - Choose MAC diet, if needed combine dietary and probiotic interventions. Ask this question: what have you fed your microbiota today? By choosing and consuming a MAC diet, you keep your gut microbiome happy; they in turn make you happy, healthy and keep you free from lifestyle diseases. More research towards a better understanding of diet-microbiota interactions will help to develop a microbiome-focused precision or personalized nutrition approach that would target and reduce more efficiently the incidence of chronic diseases. 


\section{Bibliography}

1. Balasubramanyam M. "Metagenomics Health Claim: Are you rich enough in your gut microbiota?". Biology and Medicine 6.4 (2014): 220.

2. Sonnenburg ED and Sonnenburg JL. "Starving our microbial self: the deleterious consequences of a diet deficient in microbiota-accessible carbohydrates". Cell Metabolism 20 (2014): 7.

3. Hryckowian AJ., et al. "Microbiota-accessible carbohydrates suppress Clostridium difficile infection in a murine model". Nature Microbiology 3.6 (2018): 662-669.

4. Cordain L., et al. "Origins and evolution of the Western diet: health implications for the 21st century". The American Journal of Clinical Nutrition 81 (2005): 341-354.

5. Schnorr SL., et al. "Gut microbiome of the Hadza hunter-gatherers". Nature Communications 5 (2014): 3654.

6. Sonnenburg ED., et al. "Diet-induced extinctions in the gut microbiota compound over generations". Nature 529 (2016): $212-215$

Volume 3 Issue 8 August 2019

(C) All rights are reserved by $M$ Balasubramanyam. 